\title{
Between hope and fear: patient's expectations prior to pelvic organ prolapse surgery
}

\author{
Sameh S. S. Lawndy • Mariella I. Withagen • \\ Kirsten B. Kluivers • Mark E. Vierhout
}

Received: 11 December 2010 / Accepted: 2 May 2011 /Published online: 21 May 2011

(C) The Author(s) 2011. This article is published with open access at Springerlink.com

\begin{abstract}
Introduction and hypothesis The aim of our study was to analyse the patient's expectations (fears and goals (hopes)) in women who are scheduled for pelvic organ prolapse (POP) surgery.

Methods All consecutive women awaiting surgery for POP in a tertiary urogynaecological centre were included. A short questionnaire with two open questions on goals and fears with regard to the operation was used.

Results Ninety-six out of 111 distributed questionnaires $(86 \%)$ were analysed. Goals and fears were categorized into five groups. De novo symptoms (63\%), POP recurrence $(34 \%)$ and surgical complications $(29 \%)$ were the most important fears. Symptom release (96\%), improved lifestyle (physical capabilities; 30\%) and improved sexual life (18\%) were important goals.

Conclusions A wide variety of expectations both positive and negative can be found in women before POP surgery and should be an integral part of preoperative counselling. Achieving the individual goals as based on expectations, positive (goals) and negative (fears), should be part of the POP surgery evaluation.
\end{abstract}

Keywords Pelvic organ prolapse · POP surgery · Hopes . Fears $\cdot$ Expectations

S. S. S. Lawndy $(\bowtie) \cdot$ M. I. Withagen $\cdot$ K. B. Kluivers $\cdot$

M. E. Vierhout

791 Department of Obstetrics and Gynaecology, Radboud University Nijmegen Medical Centre, P.O. Box 9101, 6500 HB Nijmegen, the Netherlands e-mail: s.lawndy@hotmail.com

\begin{abstract}
Abbreviations
POP Pelvic organ prolapse

UI Urinary incontinence
\end{abstract}

\section{Introduction}

Pelvic organ prolapse (POP) and urinary incontinence (UI) are common disorders in women and have a great impact on quality of life (QoL) [1-4]. There is an $11 \%$ lifetime risk of undergoing at least one operation for POP or UI, and there is a high prevalence of reoperations [5]. POP surgery is an important treatment for POP. There is no consensus regarding the best method of outcome assessment of POP surgery, but the achievement of patient-oriented goals gains importance [6,7]. Traditional physician-determined measures of treatment success appeared to be insufficient to determine the quality of surgical outcomes and patient satisfaction [7-9]; this may be explained by the fact that surgeons and patients had different goals and expectations on the outcomes of surgery [10]. This has led to the introduction of diseasespecific QoL questionnaires in order to determine the quality of surgical outcomes in a more patient-oriented manner [11]. But disease-specific QoL questionnaires may also lack the sensitivity to assess individual symptoms [12], and they disregard the goals of the individual patient [13-16]. Realistic and achievable goals may prevent dissatisfaction with surgical outcomes [17].

Goal achievement is now regarded as an important predictor for success after surgery. In the present study, we were specifically interested in the fears or negative expectations of our patients. Fears, justified or unjustified, may make 
patients refrain from effective treatment. In the literature, this subject has been given hardly any attention, neither in gynaecological surgery nor in the other specialties.

In this study, we summarize the patient's goals and fears before surgery in a group of women scheduled for POP surgery.

\section{Materials and methods}

Women were recruited from a tertiary urogynaecological centre in the Netherlands, the Radboud University Nijmegen Medical Centre. All consecutive women who were waiting for POP surgery during the study period from December 2008 till October 2009 were requested to complete a short questionnaire and return with a reply envelope. Outpatient counselling was performed by the outpatient gynaecologist who is in generally also the operating gynaecologist. In our study, the women were counselled by four gynaecologists who work with the same protocols and in the same group; most of the women in our study $68(71 \%)$ were counselled by one gynaecologist (MV). The preoperative counselling before the operation was not altered for this study. Counselling generally started by the referring general practitioner, but we have no information on this. Outpatient counselling consisted of counselling about the various possible therapies, a brief oral explanation of the operation supported by the handout of a standardized folder as advised by the Dutch Society for Obstetrics and Gynecology (www.NVOG.nl). Patients were standardly offered the possibility for an additional counselling consult, but this is seldom used.

The one-page questionnaire contained an introduction and two short open questions. The introduction of the questionnaire was as follows: "You are scheduled to undergo POP surgery. We are studying the reasons for undergoing such surgery. Therefore we would like to ask you to answer as accurate as possible the following two questions." The first question was "What are the most important goals you want to accomplish with the surgery? In other words what do you hope to achieve by the operation? You can, but need not necessarily, give a maximum of 5 targets, let your imagination run free!" The second question was "What are the most important things or situations that you would not want to reach by the surgery? In other words what do you fear a situation you are concerned about that could occur after the operation? You can, but need not necessarily, give a maximum of 5 things or situations. Let your imagination run free!"

The answers were collected, transferred into a database and categorized. Categorization was based on the answers and not on pre-existent categories. Simple statistical frequencies were used to describe the answers. Spearman's correlation coefficient test was used to test for potential correlations between the number of answers in the 10 categories (five hope categories and five fear categories). $P$ values $<0.05$ were considered statistically significant. Statistical analyses were performed using SPSS version 16.0 (SPSS, Inc., Chicago, IL, USA). This study was not submitted for ethics approval since the procedures and questionnaires used are part of daily clinical practice.

\section{Results}

One hundred eleven women $(n=111)$ were asked to answer our questionnaire, of whom 99 patients $(89 \%)$ replied; one of those had cancelled the operation. Two questionnaires contained no useful information, and thus, 96 questionnaires $(86 \%)$ were available for analysis.

The mean number of answers to the hope question was 2.9 (range 1-6), and the mean number of answers to the fear question was 1.8 (range $0-5$ ). The demographics and surgical procedures for the participating women are shown in Table 1, and all women included are native Dutch women. The answers to the two questions are listed in
Table 1 Demographics and surgical procedures of the study population

Data presented as number (percentage) or median (range) as appropriate

$n$ number of cases, BMI body mass index, $P O P$ pelvic organ prolapse

\begin{tabular}{ll}
\hline & POP patients $(n=111)$ \\
\hline Age $($ years $)$ & $60(23 ; 88)$ \\
BMI $\left(\mathrm{kg} / \mathrm{m}^{2}\right)$ & $27(17 ; 63)$ \\
Parity & $2(0 ; 5)$ \\
Postmenopausal (yes) & $65(68 \%)$ \\
First POP surgery (yes) & $80(83 \%)$ \\
Planned surgical procedures & \\
Anterior and/or posterior repair & $32(33 \%)$ \\
Modified Manchester with anterior and/or posterior repair & $41(43 \%)$ \\
Vaginal hysterectomy with anterior and/or posterior repair & $11(11 \%)$ \\
Abdominal sacrocolpopexy & $3(3 \%)$ \\
Prolift operations (with or without anterior and/or posterior repair) & $9(9 \%)$ \\
\hline
\end{tabular}


Tables 2 and 3, respectively. Most women (63 women, $66 \%$ ) had more goals than fears; a minority had more fears than goals (10 women, 10\%). The number of answers on goals correlated positively with the number of fears $(r=0.303$ $(P<0.01))$. Further, the hope of getting a better lifestyle category correlates positively with emotional health category $(r=0.254(P<0.05))$; the hope of getting a better sexual life category correlates positively with the fear from deterioration of sexuality category $(r=0.232(P<0.05))$.

\section{Discussion}

To our best knowledge, our study is the first on fears, besides goals, in relation to POP surgery. Fears and goals can influence decision making. Goals, certainly erroneous ones, may allow patients to go forward, with surgery when it would not be reasonable to expect those goals, but fears can also greatly influence decision making in patients and, although often heard during consultations, has been given little attention in the available literature. Our results show that besides hopes (goals), there are also a significant number of fears in the patients when specifically asked for. It was, however, notable that the number of answers regarding fears was less as compared with the answers regarding goals. The number of goals and fears were, however, positively correlated. This may be simply a result of the ability of some women to verbalize their expectations better than others

One previous study in 1993 has studied fear for cataract operations in aged persons [18]. This study has found that $33 \%$ of the patients had fear and $32 \%$ felt tension about the operation; women feared the operation significantly more than men. Fears were associated with hypochondriasis, hysteria and hypomania.

The most striking finding in our study is that almost all women feared de novo symptoms, particularly UI. This finding matches well with our clinical experience. The fear

Table 2 Categorized answers on the first question "What are your most important goals, you want to accomplish with the surgery? In other words what you hope eventually to have achieved by the operation?"

\begin{tabular}{|c|c|c|}
\hline Category & Characteristic answer & $n(\%)$ \\
\hline 1. Symptoms & & $92(96)$ \\
\hline Urinary symptoms & & $67(70)$ \\
\hline Disappearing of urine incontinence & "I do not get wet every time" & $38(40)$ \\
\hline Disappearing of urgency/frequency & "That I can walk for two hours as before without having to pee 3 times" & $26(27)$ \\
\hline Normalize micturation & "That I can pee normally as before" & $18(19)$ \\
\hline No need for sanitary napkin & "That I can go out without Tena lady (sanitary napkin)" & $10(10)$ \\
\hline Prolapse symptoms & & $50(52)$ \\
\hline Disappearing of prolapse sensation & "That no more flesh comes from my vagina" & $50(52)$ \\
\hline No more need for vaginal pessary & "No need for using pessary" & $5(5)$ \\
\hline Pain symptoms & & $29(30)$ \\
\hline Improvement of the pain (generally) & "Reduction in the abdominal pain" & $20(21)$ \\
\hline Improve back pain & "No more back pain" & $9(9)$ \\
\hline Bowel symptoms & & $20(21)$ \\
\hline Improvement of defecation & "That I can defecate normally as before" & $17(18)$ \\
\hline Disappear of faecal incontinence & "No involuntary stool loss moments" & $4(4)$ \\
\hline 2. Lifestyle & & $29(30)$ \\
\hline Improve physical capabilities & "To do my work and sport optimally without all those problems" & $29(30)$ \\
\hline 3. Sexual life & & $17(18)$ \\
\hline Improvement sexuality & "That I can have sex normally as before without hinder" & $14(15)$ \\
\hline Disappear of dyspareunia & "Sex without pain" & $3(3)$ \\
\hline 4. Emotional health & & $16(17)$ \\
\hline Improve body image & "That I become a young woman again" & $9(9)$ \\
\hline Improve fatigue symptoms & "No more feeling tired" & $8(8)$ \\
\hline 5. Other & & $6(6)$ \\
\hline Disappear of menstruation & "No more bleeding, no more periods" & $4(4)$ \\
\hline Decrease the chance to get cancer & "Prevent the risk of womb cancer, I use Tamoxifen for 4 years" & $2(2)$ \\
\hline
\end{tabular}

Data are presented as $n$ (percentage)

$n$ number of times reported 
Table 3 Answers on the second question "What are your most important things or situations that you just would not want to reach by the surgery? In other words what you want to avoid, a situation where you are concerned that could occur through the operation?"

\begin{tabular}{|c|c|c|}
\hline Category & Characteristic answer & $n(\%)$ \\
\hline 1. Getting new symptoms & & $60(63)$ \\
\hline Urine incontinence & "To become incontinent" & $50(52)$ \\
\hline Pain & "New pain complaints" & $12(13)$ \\
\hline Urine retention/incomplete urination & "That I use catheter to pee" & $9(9)$ \\
\hline Faecal incontinence & "Damage to the anal sphincter" & $5(5)$ \\
\hline 2. Recurrence & & $33(34)$ \\
\hline Failure of the surgery & "That it has not helped" & $22(23)$ \\
\hline Recurrence (soon in the future) & "That I soon get recurrence" & $14(15)$ \\
\hline 3. Surgical complications & & $28(29)$ \\
\hline Surgical complications & "That there are complications during or after the operation" & $20(21)$ \\
\hline Complications of the general/spinal anaesthesia & "I get no adverse effects after anesthesia" & $8(8)$ \\
\hline Rejection of the mesh & "I hope that no rejection of the mesh occurs" & $3(3)$ \\
\hline 4. Deterioration of sexual life & & $16(17)$ \\
\hline Getting dyspareunia & "That my vagina become narrow that sex become impossible" & $9(9)$ \\
\hline Sexually deterioration & "That sexual sense become less" & $7(7)$ \\
\hline 5. Lifestyle deterioration & & $8(8)$ \\
\hline Physical deterioration & "That hinders me in the daily activities" & $8(8)$ \\
\hline
\end{tabular}

Data are presented as $n$ (percentage)

$n$ number of times mentioned

of becoming incontinent after POP surgery may be a reason for refraining from POP surgery. The occurrence of stress UI (SUI) after POP surgery may be associated with the presence of occult stress incontinence before surgery $[19,20]$ which is estimated to be $36-80 \%$ in advanced POP [20]. The occurrence of postoperative SUI is, however, difficult to predict, and all studies on the subject show that the percentage of occult incontinence preoperatively is far higher as compared with the actual percentage of SUI after surgery [21]. The expectations of the women with regard to UI are thus somewhat discrepant with the reality. Furthermore, postoperative SUI can be managed rather effectively with tension-free mid-urethral sling operations [22]. This makes UI a crucial item for proper counselling before the operation.

In a recent study, the use of photos and videos to explain what patients could expect during and after the operation has reduced significantly preoperative anxiety [23]. Recurrence and failure after POP surgery were important issues women fear; this is more justified because the failure rate of POP surgery is high [5]. The patient's fear of surgical complications on the other hand seems rather high because the complication rates during POP surgery are in general low $[21,24]$.

The effect of POP surgery on sexuality, both fear for deterioration as well as hope for improvement, was an important subject in the women included in this study. We do not know how many women in our group were sexually active before or after the operation. A recent study reported a significant improvement in the sexual function 2 years after POP and stress UI surgery [25]. In another recent study is concluded that the POP surgery with additional incontinence surgery resulted in decreased vaginal wall sensibility; there was no influence of the decreased vaginal wall sensibility on sexual well-being [26].

In our study, we did not use a predefined system to categorize the expectations, and previous studies had used different categories $[15-17,27]$. There is no widely accepted system to categorize the goals for treatment. Lowenstein et al. [27] have categorized the goals into five categories: (1) goals related to symptom resolution, (2) goals related to lifestyle and/or quality of life improvement, (3) goals related to emotional outcome, (4) goals related to information seeking (aimed to gain more information about symptoms, treatment, or prognosis) and (5) other. Differences in goals between populations may be explained by differences in social and cultural environment. Factors such as age, culture, place and religion will affect the goals and fears of the patients who are going to be operated for pelvic floor dysfunctions.

Although it is known that POP is not a cause of pelvic or low back pain [28], 30\% of the women in our population wished resolution pain as result surgery wish may be an unrealistic aim of the surgery.

It could be expected to find that the hope to get a better sex life positively correlates with the fear from deterioration and that the hope of getting a better lifestyle correlates positively with emotional health. 
In conclusion, negative patient's expectations (fears) were present besides positive expectations (goals or hopes). Both and especially, sometimes erroneous, fears are important and should be an integral part of preoperative counselling. Further, the fears should be part of a comprehensive informed consent. Achievement of the expectations, both positive (hopes) and negative (fears), should be part of the evaluation of the outcome after surgery.

Conflicts of interest MW and MV have a consultancy agreement with and are on the Speaker's Bureau of Ethicon Women's Health \& Urology. MV is a member of the European OAB faculty of Astellas. This study, however, was entirely instigated by the responsible researchers and funded by university-administered research funds. No commercial partner was involved in the study setup, study design, data collection, or whatsoever. The other authors have no conflicts of interest to declare.

Open Access This article is distributed under the terms of the Creative Commons Attribution Noncommercial License which permits any noncommercial use, distribution, and reproduction in any medium, provided the original author(s) and source are credited.

\section{References}

1. Helstrom L, Nilsson B (2005) Impact of vaginal surgery on sexuality and quality of life in women with urinary incontinence or genital descensus. Acta Obstet Gynecol Scand 84:7984

2. Bump RC, Norton PA (1998) Epidemiology and natural history of pelvic floor dysfunction. Obstet Gynecol Clin North Am 25:723746

3. Samuelsson EC, Victor FT, Tibblin G, Svardsudd KF (1999) Signs of genital prolapse in a Swedish population of women 20 to 59 years of age and possible related factors. Am J Obstet Gynecol 180:299-305

4. Digesu GA, Chaliha C, Salvatore S, Hutchings A, Khullar V (2005) The relationship of vaginal prolapse severity to symptoms and quality of life. BJOG 112:971-976

5. Olsen AL, Smith VJ, Bergstrom JO, Colling JC, Clark AL (1997) Epidemiology of surgically managed pelvic organ prolapse and urinary incontinence. Obstet Gynecol 89:501-506

6. Mattiasson A, Djurhuus JC, Fonda D, Lose G, Nordling J, Stohrer M (1998) Standardization of outcome studies in patients with lower urinary tract dysfunction: a report on general principles from the Standardisation Committee of the International Continence Society. Neurourol Urodyn 17:249-253

7. Barber MD, Brubaker L, Nygaard I, Wheeler TL, Schaffer J, Chen Z, Spino C (2009) Defining success after surgery for pelvic organ prolapse. Obstet Gynecol 114:600-609

8. Robinson D, Anders K, Cardozo L, Bidmead J (2007) Outcome measures in urogynaecology: the clinicians' perspective. Int Urogynecol J Pelvic Floor Dysfunct 18:273-279

9. Lawndy SS, Kluivers KB, Milani AL, Withagen MI, Hendriks JC, Vierhout ME (2010) Which factors determine subjective improvement following pelvic organ prolapse 1 year after surgery? Int Urogynecol J Pelvic Floor Dysfunct 22(5):543-549
10. Srikrishna S, Robinson D, Cardozo L (2010) A longitudinal study of patient and surgeon goal achievement 2 years after surgery following pelvic floor dysfunction surgery. BJOG 117:1504-1511

11. Bovbjerg VE, Trowbridge ER, Barber MD, Martirosian TE, Steers WD, Hullfish KL (2009) Patient-centered treatment goals for pelvic floor disorders: association with quality-of-life and patient satisfaction. Am J Obstet Gynecol 200:568.e1-568.e6

12. Srikrishna S, Robinson D, Cardozo L, Cartwright R (2008) Experiences and expectations of women with urogenital prolapse: a quantitative and qualitative exploration. BJOG 115:1362-1368

13. Cammu H, Van Den Abbeele E, Nagel H, Haentjens P (2009) Factors predictive of outcome in tension-free vaginal tape procedure for urinary stress incontinence in a teaching hospital. Int Urogynecol J Pelvic Floor Dysfunct 20:775-780

14. Hullfish KL, Bovbjerg VE, Gibson J, Steers WD (2002) Patient-centered goals for pelvic floor dysfunction surgery: what is success, and is it achieved? Am J Obstet Gynecol 187:88-92

15. Brubaker L, Shull B (2005) EGGS for patient-centered outcomes. Int Urogynecol J Pelvic Floor Dysfunct 16:171-173

16. Elkadry EA, Kenton KS, FitzGerald MP, Shott S, Brubaker L (2003) Patient-selected goals: a new perspective on surgical outcome. Am J Obstet Gynecol 189:1551-1557

17. Mahajan ST, Elkadry EA, Kenton KS, Shott S, Brubaker L (2006) Patient-centered surgical outcomes: the impact of goal achievement and urge incontinence on patient satisfaction one year after surgery. Am J Obstet Gynecol 194:722-728

18. Fagerstrom R (1993) Fear of a cataract operation in aged persons. Psychol Rep 72:1339-1346

19. Liang CC, Chang YL, Chang SD, Lo TS, Soong YK (2004) Pessary test to predict postoperative urinary incontinence in women undergoing hysterectomy for prolapse. Obstet Gynecol 104:795-800

20. Haessler AL, Lin LL, Ho MH, Betson LH, Bhatia NN (2005) Reevaluating occult incontinence. Curr Opin Obstet Gynecol 17:535-540

21. Caquant F, Collinet P, Debodinance P, Berrocal J, Garbin O, Rosenthal C, Clave H, Villet R, Jacquetin B, Cosson M (2008) Safety of Trans Vaginal Mesh procedure: retrospective study of 684 patients. J Obstet Gynaecol Res 34:449-456

22. Groutz A, Levin I, Gold R, Pauzner D, Lessing JB, Gordon D (2010) "Inside-out" transobturator tension-free vaginal tape for management of occult stress urinary incontinence in women undergoing pelvic organ prolapse repair. Urology 76:13581361

23. Gonzales EA, Ledesma RJ, McAllister DJ, Perry SM, Dyer CA, Maye JP (2010) Effects of guided imagery on postoperative outcomes in patients undergoing same-day surgical procedures: a randomized, single-blind study. AANA J 78:181-188

24. Lo TS (2010) One-year outcome of concurrent anterior and posterior transvaginal mesh surgery for treatment of advanced urogenital prolapse: case series. J Minim Invasive Gynecol $17: 473-479$

25. Srikrishna S, Robinson D, Cardozo L, Gonzalez J (2010) Can sex survive pelvic floor surgery? Int Urogynecol J Pelvic Floor Dysfunct 21:1313-1319

26. Lakeman MM, van der Vaart CH, Laan E, Roovers JP (2011) The effect of prolapse surgery on vaginal sensibility. J Sex Med $8: 1239-1245$

27. Lowenstein L, FitzGerald MP, Kenton K, Dooley Y, Templehof M, Mueller ER, Brubaker L (2008) Patient-selected goals: the fourth dimension in assessment of pelvic floor disorders. Int Urogynecol J Pelvic Floor Dysfunct 19:81-84

28. Heit M, Culligan P, Rosenquist C, Shott S (2002) Is pelvic organ prolapse a cause of pelvic or low back pain? Obstet Gynecol 99:23-28 